\title{
The Hyper-trapeze: A Physically Active Audio-Visual Interface for Performance and Play
}

\author{
Anne Hoekstra ${ }^{1}$, Christoph Bartneck ${ }^{1}$, and Michael J. Lyons ${ }^{2}$ \\ ${ }^{1}$ Eindhoven University of Technology, Department of Industrial Engineering \\ a.hoekstra@student.tue.nl, c.bartneck@tue.nl \\ ${ }^{2}$ Ritsumeikan University, College of Image Arts and Sciences \\ lyons@im.ritsumei.ac.jp
}

\begin{abstract}
This paper reports the design, implementation, and application of a new interface for augmenting performance and play on the low flying trapeze. Sensors were used to gauge the motion of the trapeze and performer, mapping the data to control interactive sound and animation. The interface was tested in the context of a new multimedia composition entitled "Autumn".
\end{abstract}

Keywords: trapeze, performance interface, exertainment, exertion interface.

\section{Introduction}

Recently there is growing interest in the field of exertainment, which combines physical activity with interactive entertainment [1]. Interactive entertainment technology is being adopted in physical education settings to motivate exercise by offering players fun and novelty [2]. In this project we extend this concept to artistic and expressive play, using a performance medium, the low-flying trapeze, which is used by some modern dancers. We created a novel system that responds to the movements of the player to produce sound and animated graphics. Our intention is twofold: first to create a new performance interface, which we call the hyper-trapeze, which bridges the digital and physical world by augmenting an existing technology, the low-flying trapeze, with interactive multimedia. Second, to explore methods of interacting with the hypertrapeze, both for purposes of artistic performance and for enjoyable physical activity.

\section{Trapeze Background}

The main inspiration for this project is the low flying trapeze, shown in figure 1 , a performance technology used in some forms of contemporary dance. We were introduced to the low-flying trapeze by dancer/choreographer Peter GoLightly, of the Kyoto KyoRyuKan studio. In contrast to its circus cousin, the high-flying trapeze, it does not require a performer to risk life and limb: it hangs just high enough the ground to allow spinning, swinging, with legs airborne, and dance maneuvers with the feet on the ground. By allowing the dancer to leave the ground, and spin at high angular velocity, the trapeze expands the range of space and movement that may be 


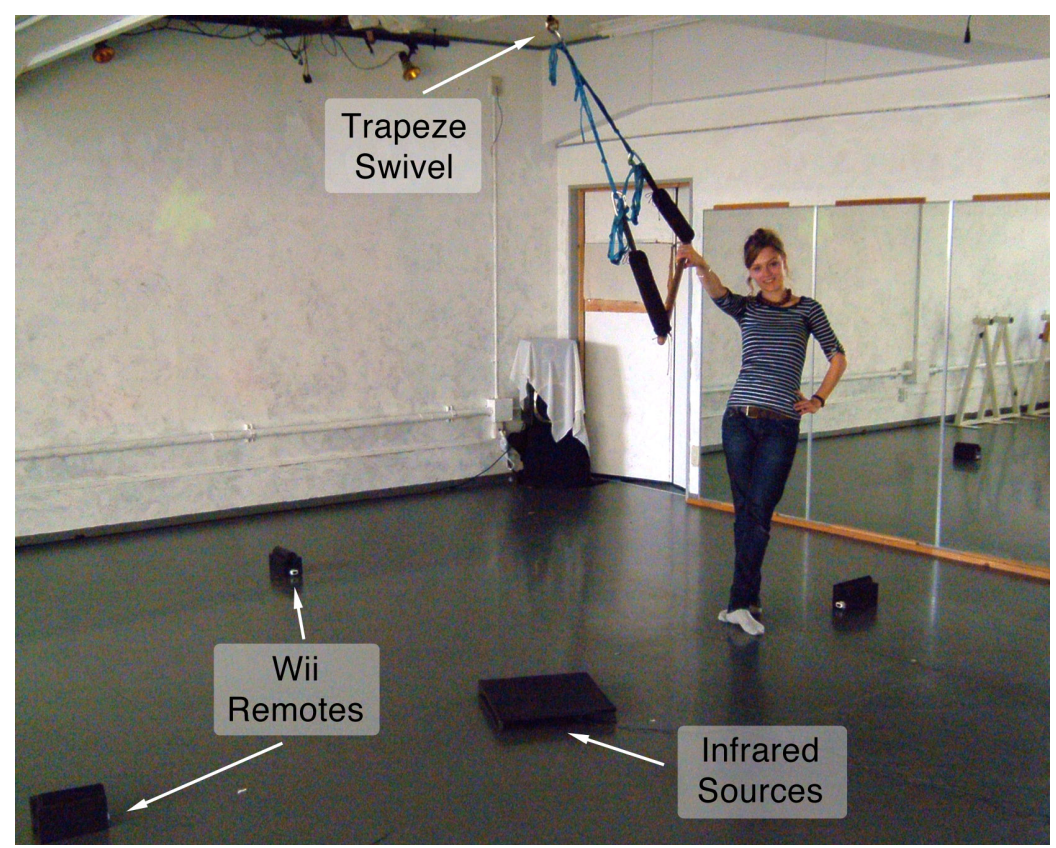

Fig. 1. Overview of the hyper-trapeze system

used in a performance. This can greatly enhance the drama, variety, and excitement of a modern dance performance. At the same time, the novel appearance and perceived affordances of the trapeze invite play. With a soft studio floor, the trapeze is no more dangerous than a playground swing. These qualities of the trapeze suggest that, in addition to its role in performance, it may be suitable for use in exergaming.

The physical design of the low-flying trapeze is fairly straightforward. Unlike the circus trapeze, a wooden bar is suspended from a single point by a swivel attached to the ceiling with ropes, and a carabiner attached to the trapeze straps, allowing the user to use the same swivel with different trapezes. Soft grips are attached to the straps immediately adjacent to the wooden trapeze bar protecting the performer from rope burns.

The swivel allows the trapeze to spin without the support ropes twisting together; it must also safely support the performer's weight and spin freely. We used a Petzl P58 swivel [3]. This swivel is used by mountaineers when rappelling as well as in rescue work, and has been individually tested for a rated working load limit of $5 \mathrm{kN}$ (kilonewtons), with a minimum breaking strength of $36 \mathrm{kN}$, more than sufficient to safely support an individual.

\section{Hyper-trapeze Design Considerations}

The trapeze acts passively to allow the performer a greater range of expressive movement. As a physical object responsive to the intentions of the dancer, the trapeze presents opportunities to interface with interactive digital media in a non-encumbering 
fashion. We asked whether augmenting the trapeze with sensors could allow it to become an audio-visual instrument, expanding the performers expressive scope.

This inspiration came from observing trapeze performances. To ground our design in expertise, we interviewed a trapeze performer (PG) about his approach to choreography and performance with the trapeze, and also carefully observed the trapeze while he demonstrated typical movements. To summarize, a lot of spinning movements are made, with the performer moving in a circular area centered below the swivel. The motion of the swivel itself is normally circular or linear depending on whether the trapeze is spinning or swinging. It can also be stationary when the performer climbs on the straps, or dances without the trapeze. PG also explained to us that he often moves his feet on the floor in painting movements in the circular area below the swivel and expressed a strong wish to have sensors responsive to these patterns. Our discussions produced a set of design requirements for the hyper-trapeze: the system should respond to the performer's intentions; the added technology must not interfere with the safety, or comfort of the trapeze; the system must be robust to vigorous movement; the design should be trapeze specific; and the development time required must be reasonable.

Based on these discussions we sketched several ideas for sensing movement of the trapeze and performer. Because the swivel is fairly isolated from the rest of the assembly and moves smoothly even for vigorous performances, we thought about various ways to sense its motion, including optical tracking of markers on the swivel, and magnetic tracking via a Hall effect sensor. Similarly, the ceiling support ropes are stationary during performance but experience variable load, leading us to consider force sensitive resistors (FSR) as a means to sense the shifting load and acceleration of the performer. We also considered directly sensing the dancer's body or the trapeze bar via optical tracking and reflective markers, finally, following the dancer's request, we considered adding sensors to the floor to allow the dancer to trigger effects with the feet. Here two alternatives were considered: tripping an optical switch with a laser beam and light sensor, or a similar arrangement but using the near infrared camera in

Table 1. Evaluation of design proposals for fulfillment of requirements $(0-$ low; $2-$ high $)$

\begin{tabular}{|c|c|c|c|c|c|c|c|c|}
\hline $\begin{array}{c}\text { Sensed } \\
\text { Variable }\end{array}$ & Sensor & Control & Safety & $\begin{array}{c}\text { Robust- } \\
\text { ness }\end{array}$ & Comfort & $\begin{array}{c}\text { Trapeze } \\
\text { Specific }\end{array}$ & Time & Total \\
\hline $\begin{array}{c}\text { Swivel } \\
\text { Motion }\end{array}$ & Camera & 1 & 2 & 1 & 2 & 2 & 1 & 9 \\
\cline { 2 - 9 } & Hall Effect & 1 & 2 & 1 & 2 & 2 & 1 & 9 \\
\hline $\begin{array}{c}\text { Strap } \\
\text { Pressure }\end{array}$ & $\begin{array}{c}\text { Force } \\
\text { Sensitive } \\
\text { Resistors }\end{array}$ & 1 & 2 & 2 & 2 & 2 & 2 & 11 \\
\hline $\begin{array}{c}\text { Body } \\
\text { Motion }\end{array}$ & Camera & 1 & 2 & 1 & 1 & 0 & 0 & 5 \\
\hline $\begin{array}{c}\text { Trapeze } \\
\text { Motion }\end{array}$ & Camera & 1 & 1 & 1 & 0 & 1 & 0 & 4 \\
\hline $\begin{array}{c}\text { Foot } \\
\text { Position }\end{array}$ & Laser Beam & 2 & 2 & 0 & 2 & 2 & 2 & 10 \\
\cline { 2 - 9 } & Wii Remote & 2 & 2 & 2 & 2 & 2 & 2 & 12 \\
\hline
\end{tabular}


the Wii remote with IR LEDs as light sources. Since this beam trigger resembles the famous Jean-Michel Jarre's famous "Laser Harp" musical interface, we call this a "Floor Harp". We evaluated the many ideas generated in terms of the design requirements listed above, with three score levels: $\mathbf{0}$ - the idea does not fit the requirement; $\mathbf{1}$ - the idea does fit the requirement; and $\mathbf{2}$ - the idea fits the requirement very well. Table 1 summarizes the idea evaluation process leading to the selection of the design elements to be implemented.

\section{Hyper-trapeze Implementation}

Based on an evaluation of the design concepts (Table 1), we implemented a prototype hyper-trapeze by eliminating low-scoring design ideas. The chosen design has: (a) a Hall effect sensor to gauge the motion of the trapeze swivel (b) two force sensors placed under the support straps and (c) a four "string" Floor harp with four Wii motes and four clusters of IR LEDs. Sensor data was acquired at approximately $200 \mathrm{~Hz}$ and sent wirelessly to a computer running Max/MSP [4] via a Bluetooth I-CubeX A/D convertor and microcontroller [5]. The Max/MSP patch also processed raw sensor data from the Wii motes and I-CubeX in real-time mapping the output to control sound synthesis. The Max/MSP patch sent data via a Flash server plug-in to a realtime Flash animation that was projected onto a wall adjacent to the trapeze. Small but

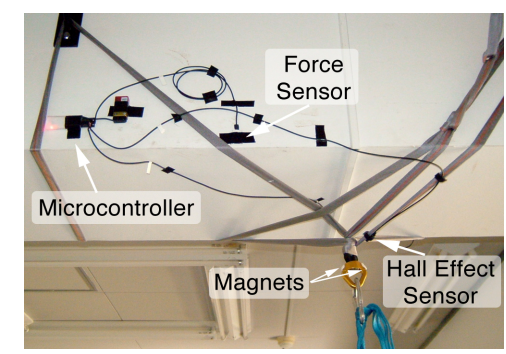

Fig. 2. Swivel motion and rope force sensors

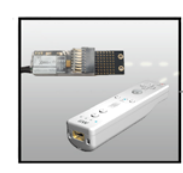

Wii-motes \& I-Cube

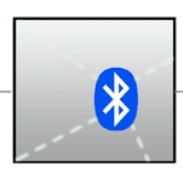

Bluetooth

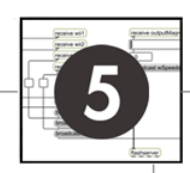

Max MSP

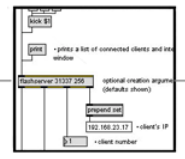

FlashServer

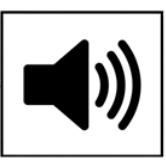

Speakers

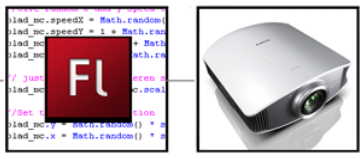

Flash

Projector

Fig. 3. System architecture of the hyper-trapeze 
powerful neodymium magnets mounted on the swivel generate a signal in the Hall effect sensor which varies with the distance from the magnet; the maximum and minimum of the signal corresponding to the closest and furthest separations of the magnet and sensor. Analysis of this signal allows us to gauge the movement of the trapeze. The force sensors are calibrated to trigger events when a threshold is exceeded by pulling heavily on the straps.

\section{Application of the Hyper-trapeze}

Interactive audio-visual contents used with the hyper-trapeze interface were developed with a play/performance environment in mind: we chose the theme of "Autumn" inspired by the beautiful changing leaves of Kyoto's maple trees. The interactive animation shows falling coloured leaves blown here and there by the wind, while interactive Max/MSP patches play sounds of the blowing wind, raindrops and puddle splashes. The mapping from sensor signals to interactive effects was chosen to be simple, direct, and transparently understandable by novice players and spectators. The choice of mapping was informed both by discussion with a trapeze expert, and by many works presented at the NIME conferences [6] where the issue of performance interface sensor to output mapping has been studied. A review of musical interface design is beyond the scope of this paper, but we recommend two excellent foundational works $[7,8]$. Briefly, the simple mapping we chose is oriented towards novices rather than virtuoso performers, as is appropriate for a completely new interface. The mapping is as follows: blocking a floor harp IR beam triggers playback of a water drop or splash and adds a leaf to the animation; rotation of the trapeze controls the cutoff frequency of resonant low-pass filtered noise, giving a remarkably convincing simulation of blowing wind. Spinning the swivel faster makes the wind blow harder both in the simulated sound and animation. Rotation direction is mapped to wind direction in the animation. Pulling a strap hard triggers bass notes or Japanese kana syllables, but is not currently mapped to visual output.

\section{Related Work}

Many prior works, too numerous to list here, have augmented dance performance with interactive audio and visual content. Two exemplary works are those by Winkler [7] and Camurri et al. [8]. In both, and most work in this area, cameras, or motion sensors capture movement of the dancers body. In contrast, in addition to having developed the world's first digitally augmented trapeze, we have taken a quite different approach in sensing the motion of a physical object other than the dancer's body. The hyper-trapeze is therefore akin to a musical instrument in that it is an object that responds to the conscious intentions and action of the player.

\section{Conclusion}

In collaboration with a dancer/choreographer we have designed and implemented a new interface for augmenting performance and play on the low-flying trapeze with 
interactive audio and animation. The first application of this system was a multimedia composition based on the theme "Autumn", but the system is flexible and can be adapted to offer new opportunities for creative expression by choreographers and dancers. We are also interested in the application of the hyper-trapeze to environments for fun, creative, and physically active play by both children and adults and we hope that this first study will facilitate future work in this direction.

\section{Acknowledgement}

This research was supported in part by a grant from the Japan Society for the Promotion of Science.

\section{References}

1. Brown, D.: Playing to Win: Video Games and the Fight against Obesity. Journal of the American Dietetic Association 106(2), 188-189 (2006)

2. Computer dance gets pupils active. BBC Online News, September 15 (2007)

3. Petzl Official website, http: / /www. petzl. com (accessed January 30, 2009)

4. Max/MSP, http: / /www. cycling74 . com/ (accessed January 30, 2009)

5. I-CubeX Wireless microsystem, http://infusionsystems.com/ (accessed January 30, 2009)

6. Poupyrev, I., Lyons, M.J., Fels, S., Blaine, T.: New Interfaces for Musical Expression. Extended Abstracts. In: Conference on Human Factors in Computing Systems (CHI 2001), pp. 491-492 (2001)

7. Cook, P.R.: Principles for Designing Computer Music Controllers. In: Proceedings, New Interfaces for Musical Expression (NIME 2001) (2001)

8. Verplank, B.: A Course on Controllers. In: Proceedings New Interfaces for Musical Expression (NIME 2001) (2001)

9. Winkler, T.: Fusing Movement, Sound, and Video in Falling Up, an Interactive Dance/Theatre Production. In: Proceedings, International Conference on New Interfaces for Musical Expression (NIME 2002), pp. 188-189 (2002)

10. Camurri, A., Hashimoto, S., Ricchetti, M., Ricci, A., Suzuki, K., Trocca, R., Volpe, G.: EyesWeb: Toward Gesture and Affect Recognition in Interactive Dance and Music Systems. Computer Music Journal 24(1), 57-69 (2000) 